\title{
Automatic Syringe for Farm Animals Vaccination
}

\author{
Vitaly Grinchenko*, Gennady Nikitenko, Evgeny Konoplev, Pavel Konoplev, Alexander Yanovskiy and Igor Devederkin
}

Stavropol State Agrarian University, Russia

\begin{abstract}
Existing injectors use the manual, mechanical or pneumatic drive. These types of drive do not allow making mass vaccination of farm animals automatic. In case of large livestock herd, vaccination is a labour intensive and complicated process. In the livestock sector, mass vaccination is carried out with intervals from several days to several months and depends on the type of animals, their breed and age. If vaccination is not provided or done incorrectly; then the weight gain of animals decreases, and the loss of livestock increases, which causes direct losses to farmers and to livestock enterprises. Therefore, special attention should be given to animals vaccinations, and scientific and technical developments for the vaccination process improvement are relevant worldwide. The developed automatic injector is a stand-alone small hand electric tool. It has an ergonomic design with a convenient location in the hand of a veterinarian and allows you to automate the process of farm animals vaccinations. A distinctive feature of the developed automatic injector is the use of linear electric motor which moves the syringe without the efforts from a veterinarian. The electric drive expands the functionality of the injector, makes the vaccination process less costly and more informative for the veterinary services using RFID identification of farm animals.
\end{abstract}

\section{Introduction}

The vaccination of farm animals is necessary for their protection from diseases and aimed at intensive growth. In animal husbandry, mass vaccination is carried out with intervals from several days to several months, depending on the type of farm animals and their age. The vaccination is a time-consuming and difficult process when dealing with a large population. During this veterinary procedure, various injection devices greatly facilitate the work of the veterinarian, reduce the possible errors, improve the quality and safety of vaccination.

If vaccination is not carried out or done incorrectly; the weight gain of animals decreases and the loss of livestock increases. This causes direct losses to farmers and livestock enterprises. In addition to the correct application of vaccination, a veterinarian should keep the records and control the doses of substances for injection. Therefore, special attention should be given to the vaccination of animals, and the development of new designs of devices for vaccination is a vital task.

\section{Materials and methods}

A huge number of manufacturers all over the world produce a wide range of injection devices, the main difference from which is the type of plunger rod drive (Figure 1).

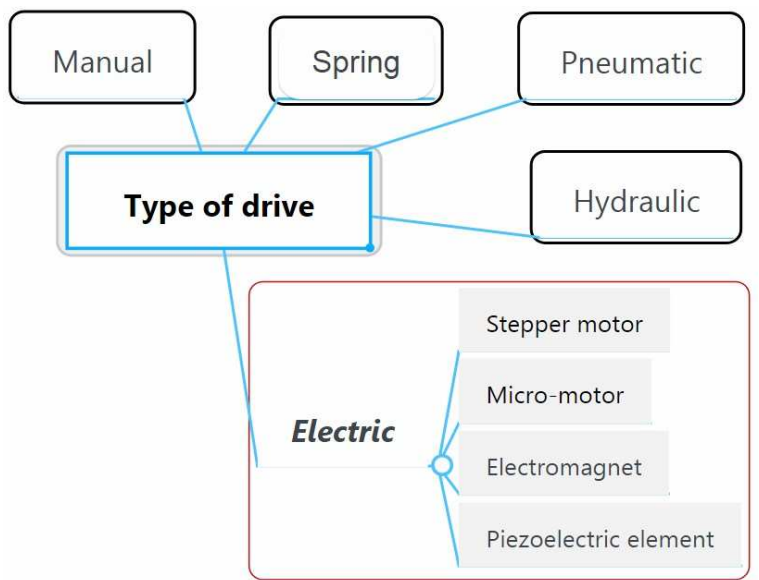

Fig. 1. The classification of devices for injecting farm animals by the type of the plunger rod drive

Vaccination is done with special technical devices; the details of their design evolution are described by Norn S., Wadham P. et al. [1, 2]. Their works conclude that the evolution of the syringe and its needle continues with the introduction of transdermal drug delivery by micron-scale needles and monitored drug delivery. The injection device with manual drive is the simplest and most wide-spread device. The principle of operation of the injection device with the manual drive is based on the application of physical force of the operator. This type of the device includes various veterinary syringes (Figure 2). From the point of view of the complexity of using such devices, its application is desirable for a single vaccination of an individual animal.

\footnotetext{
* Corresponding author: grinchen_ko@mail.ru
} 


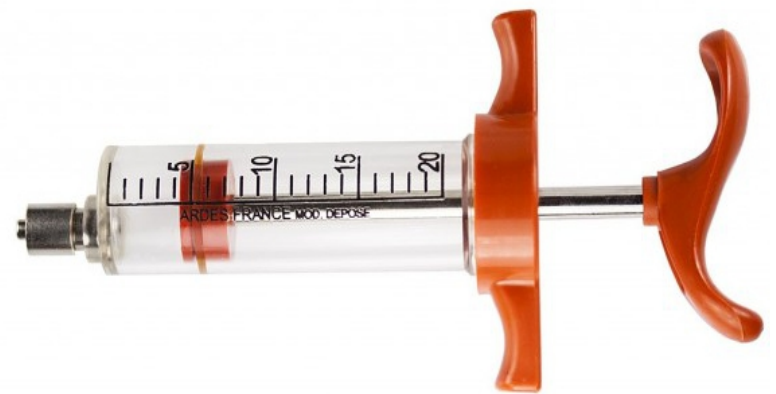

Fig. 2. Reusable veterinary syringe "Ardes" (France)

Also, the researchers dealing with the improvement of injection devices design note that devices that are easy to use and require no extra effort on behalf of the healthcare worker are preferred [3]. The spring-driven injection devices feature more advanced designs that reduce or eliminate the physical effort of the operator (Figure 3). The cylindrical screw compression springs made of steel wire with elastic properties, torsion springs or watch springs (known as feed springs) of different types are used to create the force. The sharp deformation of the springs makes the animal feel a heavy blow when the injection is initiated.

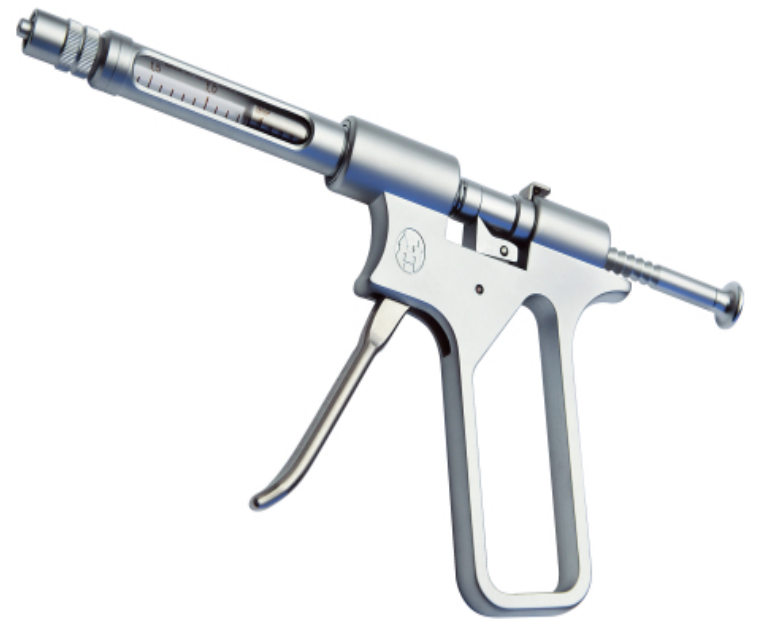

Fig. 3. Dosing syringe "HSW HENKE-JECT" (Germany)

The pneumatic and hydraulic drives of the plunger rod in injection devices (Figures 4,5 ) require additional equipment to maintain the pressure of the drive mechanism (pumps, various tanks, tube system), which limits the mobility of the operator during vaccination of animals. Since such devices have a spring drive, the control of the smooth movement of plunger rod is a challenging task.

The most promising drive in terms of functionality and possibility of automation of the process of farm animals vaccination is the electric drive of the plunger rod in devices for vaccination. The works of Ong J., Chung D., Lin E., Abid H., Liew O., $\mathrm{Ng} \mathrm{T.,}$ Jafarzadeh M., Farokhi F., Saidi I., El L., Ounip A. and Benrejeb M. recommend using the ultra-small stepper motor [4-6]. A prototype pump actuated by a piezoelectric stack actuator is developed and a PID feedback controller is designed for the drive of the injection device [7]. The electric drive in the injector devices allows creating high precision medical and veterinary devices with control based on microcontroller [8] or Rasberry $\mathrm{Pi}$ minicomputer [9], or even advancements in wireless technologies and internet of things (IoT) [10]. However, in existing devices, the drive of the plunger rod is carried out by stepper motors, micro-motors, electromagnets or piezoelectric elements that do not directly provide linear reciprocating movement of the plunger rod. For this purpose, additional gear mechanisms are used, which provide a more complicated design and reduce the reliability of the device as a whole.
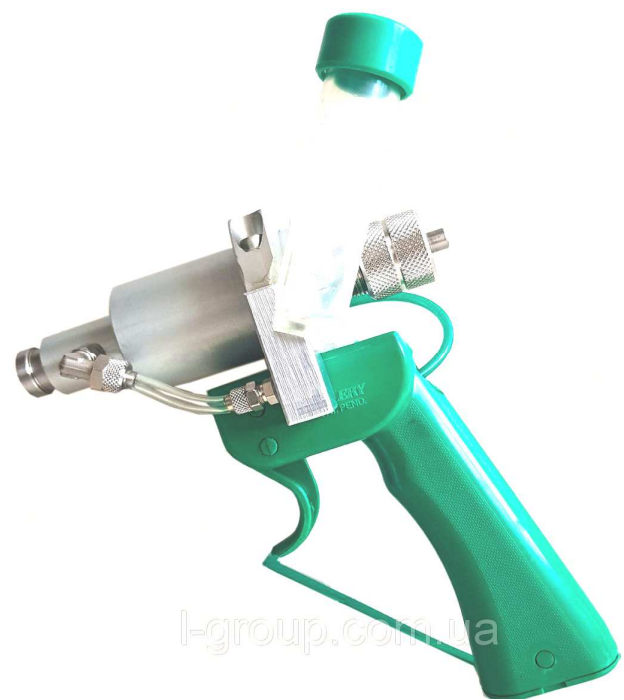

Fig. 4. Pneumatic syringe for vaccination (Italy)

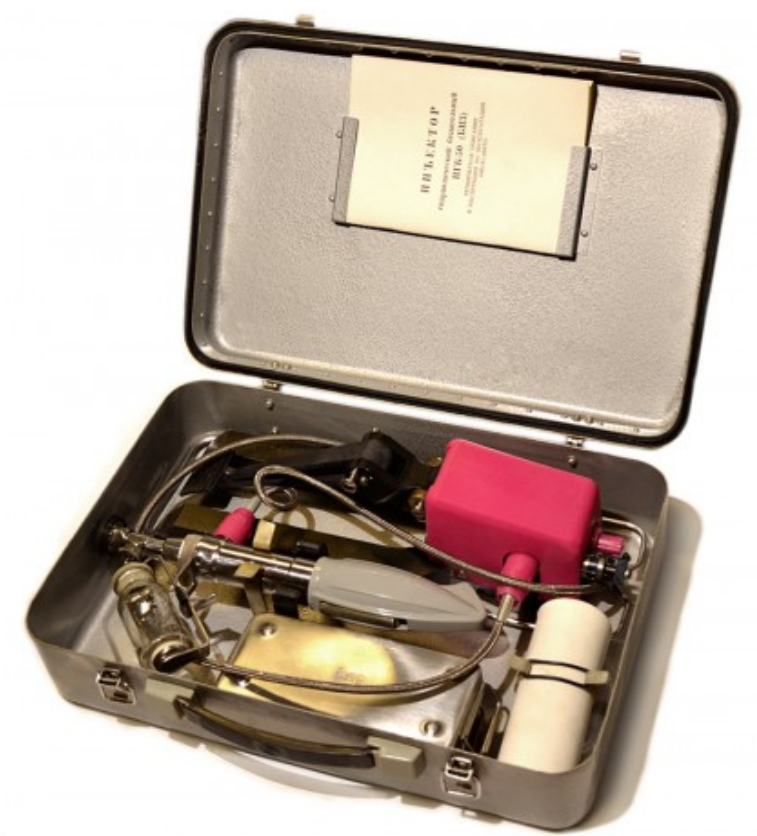

Fig. 5. "BI-3" Hydraulic injector (Russia)

\section{Results and discussion}

The analysis of the principle of operation of injection devices reveals four typical stages of linear movement of the plunger rod, and these stages of movement occur along one axis: 
- Stage I - direct movement of the needle to pierce the body of an animal;

- Stage II - direct movement of the rod to squeeze the medicine;

- Stage III - reverse movement of the rod to adjust the volume of the next dose of the medicine;

- Stage IV - reverse movement to remove the needle from the body of the animal.

All four typical stages of linear movement of the plunger rod can be realized by a linear motor. In addition, the linear motor allows to adjust the duration of the injection process, control the depth of needle penetration, vary the volume of the administered dose of the medicine, reverse the needle after injection, automatically fill the syringe with the next dose of the medicine. For these purposes, the Department of Electricity Application in Agriculture of Stavropol State Agrarian University has developed a new design of the linear motor (Figure 6) [11]. It consists of two cylindrical magnetic cores 5 with magnetizing coils 4 and 7 . In the axial hole of the magnetic cores 5 , a rod 3 is inserted, on which an anchor 6 is fixed. The anchor 6 consists of two magnetic disks 1 and non-magnetic layer 2 .

The developed linear motor works as follows. When you turn on the coil 7, the current flowing in it induces a magnetic field, the power lines of which are closed with the magnetic core 5 and the magnetic insert 1 . In this case, there is a force that moves the anchor 6 up. The force at the anchor 6 is transmitted through the rod 3 of the plunger of the injector. An increase in the current on the coil 7, provides an increase in the speed of movement of the anchor 6 . The current flowing to the coil 4 provides a force which moves the anchor 6 down. The increase in current on the coil 4 disturbs the balance between the forces, and the anchor 6 moves down.

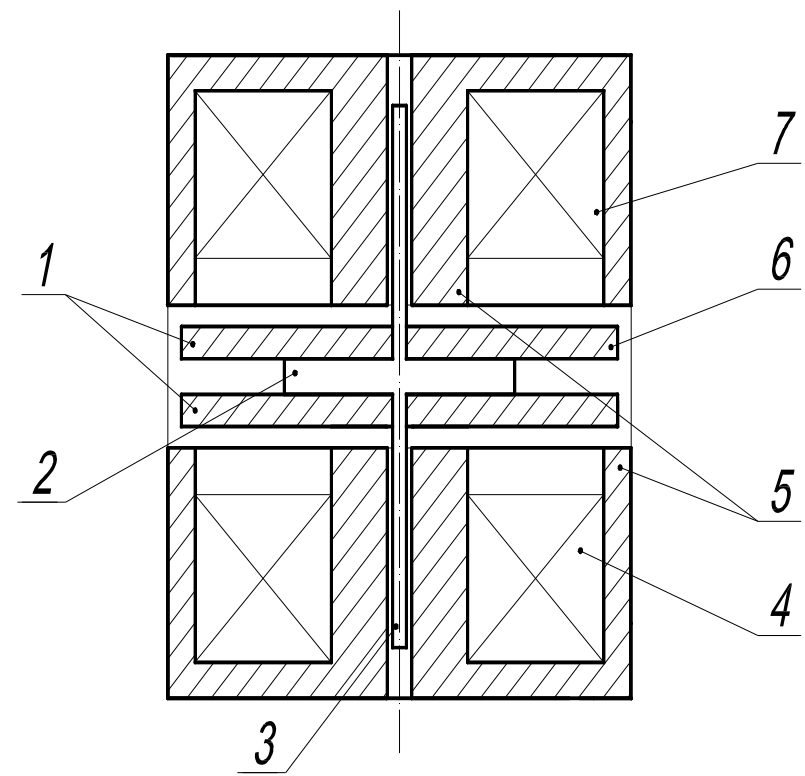

Fig. 6. Design of the developed linear electric motor for the drive rod plunger device for injection of farm animals

Thus, feeding the coils 4 and 7 with a certain value of current can change the position of the anchor 6. By setting the dynamics of the anchor movement, it is possible to control the movement of the plunger of the injector. The position of the anchor 6 is monitored by the control unit by determining the current in the coils 4 and 7. Power coils 4 and 7 are carried out using pulse width modulation, the average value of the current in them is determined by the pulse width modulation duty cycle. Changes in the duty cycle lead to a change in the average current in the coils. To achieve smooth and predictable movement of the linear motor armature under the action of the magneto-motive force, two negative feedback loops are organized in the control unit circuit. An indirect parameter of the current position of the armature is the effective value of the current flowing in the coils of the linear motor. Thus, the developed design of the linear motor allows one to realize four typical stages of movement of the plunger rod of devices for injection of farm animals and to create an essentially new device for injections (Figure 7).
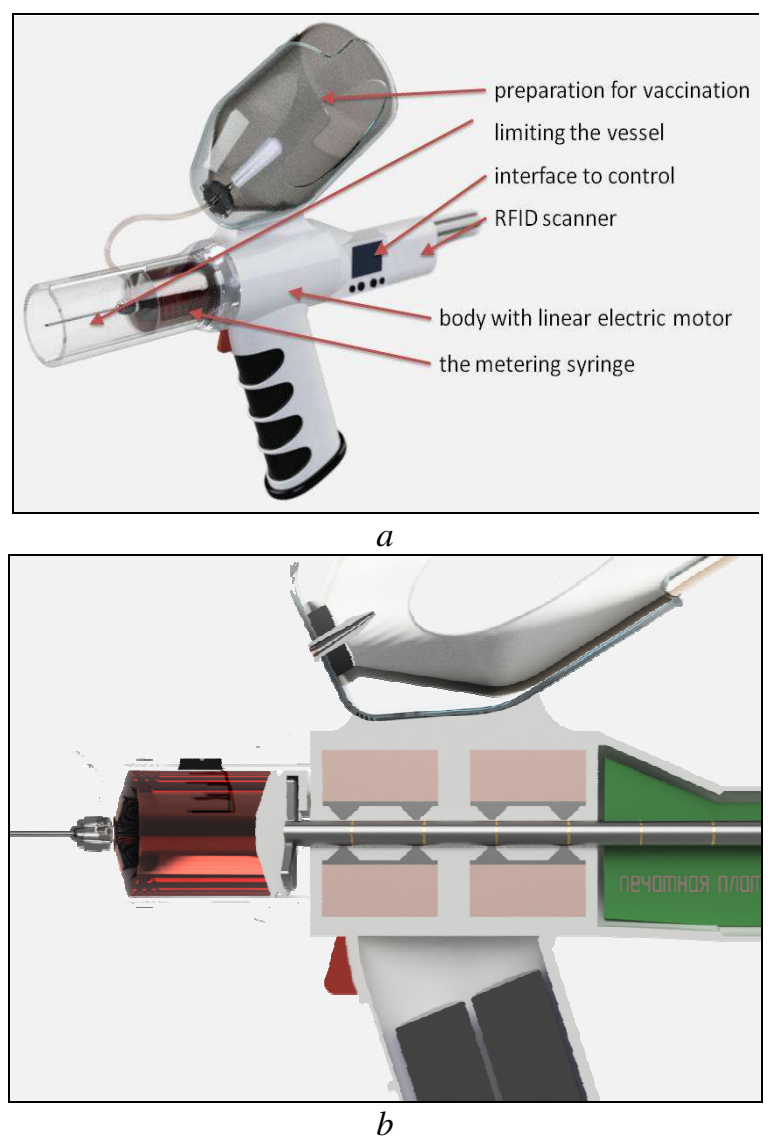

Fig. 7. The device developed for vaccination of farm animals: $\mathrm{a}$ - general form, $\mathrm{b}$ - body with linear electric motor

The calculation of the linear motor magnetic system is made by the finite-element method in the Elcut software package, which starts with the partition of the linear motor magnetic system into finite elements (Fig. 8).

The Poisson equation, which describes the magnetic state as partial derivatives in cylindrical coordinates $\mathrm{r}, \mathrm{z}$, is as follows [12]:

$$
\frac{\partial}{\partial r}\left(v \frac{1}{r} \frac{\partial}{\partial r}(r A)\right)+\frac{\partial}{\partial z}\left(v \frac{1}{r} \frac{\partial}{\partial z}(r A)\right)=J
$$



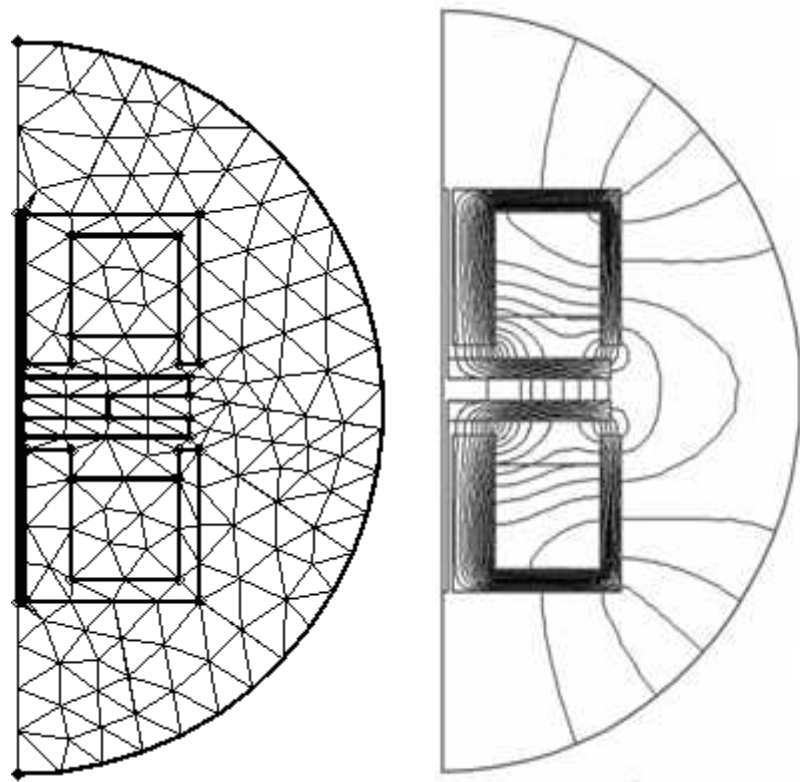

Fig. 8. Magnetic system of the designed linear electric motor

To solve the equation, the variations calculus is used, which allows applying of the energy method based on replacing the boundary problem for the Poisson equation by the problem of minimum energy functional:

$$
F=\iint_{\Omega}\left[\frac{1}{2} v\left(\frac{\partial^{2} A}{\partial z^{2}}+\frac{1}{r^{2}} \frac{\partial^{2}(r A)}{\partial r^{2}}\right)-J A\right] d \Omega,
$$

where $d \Omega=2 \pi r d r d z$.

Minimization of this equation allows defining the magnetic vector potential within the $\Omega$ area. The components of the magnetic induction vector are $B_{\Gamma}$ and $B_{Z}$ :

$$
\begin{gathered}
B_{r}=-\frac{\partial A^{\ell_{i}}}{\partial z}=-\frac{1}{2 S}\left(c_{i} A_{i}^{\ell_{i}}+c_{j} A_{j}^{\ell^{{ }_{i}}}+c_{k} A_{k}^{{ }^{\ell_{i}}}\right), \\
B_{z}=\frac{\partial A^{\ell_{i}}}{\partial r}+\frac{A^{\ell_{i}}}{r}= \\
=\frac{1}{2 S}\left[\begin{array}{l}
\left(b_{i} A_{i}^{\ell_{i}}+b_{j} A_{j}^{\ell_{i}}+b_{k} A_{k}^{\ell_{i}}\right)+ \\
+\frac{3}{r_{i}+r_{j}+r_{k}}\left(\begin{array}{l}
\left(a_{i}+b_{i} r_{i}+c_{i} z_{i}\right) A_{i}^{\ell_{i}}+ \\
+\left(a_{j}+b_{j} r_{j}+c_{j} z_{j}\right) A_{j}{ }^{\ell_{i}}+ \\
+\left(a_{k}+b_{k} r_{k}+c_{k} z_{k}\right) A_{k}^{\ell_{i}}
\end{array}\right)
\end{array}\right]
\end{gathered}
$$

where $A^{\ell} i_{i}$ is a magnetic force vector in the finite element; $S$ is an area of the finite element.

The indexes $a, b$ and $c$ in the equations (3) and (4):

$$
\begin{aligned}
& a_{i}=r_{j} z_{k}-r_{k} z_{j} ; b_{i}=z_{j}-z_{k} ; c_{i}=r_{k}-r_{j} ; \\
& a_{j}=r_{k} z_{i}-z_{k} r_{i} ; b_{j}=z_{k}-z_{i} ; c_{j}=r_{i}-r_{k} ; \\
& a_{k}=r_{i} z_{j}-r_{j} z_{i} ; b_{k}=z_{i}-z_{j} ; c_{k}=r_{j}-r_{i} .
\end{aligned}
$$

The magnetic induction vector inside the triangular element is calculated by the formula:

$$
B^{\ell_{i}}=\sqrt{B_{r}^{2}+B_{z}^{2}} \text {. }
$$

The force at the armature is defined by the equation:

$$
F=\frac{\pi D_{K}^{2}}{8 \mu_{C T} \mu_{O}} B
$$

The obtained magnetic induction value allows creating a force at the linear electric motor armature sufficient to move the injector operating mechanism and to perform the operational procedures. At this point of time, intellectual property rights are being registered, the design of the injector is not disclosed in detail, but the experience of developing such devices allows us to conclude about the distinctive features of the new design. In comparison with the existing analogues, the developed automated injector has the following advantages:

1. Automatic recording of the date of vaccination and the injection volume.

2. Automatic adjustment of the dose of the injected substance.

3. Automatic selection of type of injection (subcutaneous or intramuscular).

4. Vaccination of farm animals with one hand without the physical efforts of a veterinarian.

5. High accuracy of dosing.

6. Reduction of time of livestock mass vaccination.

In existing designs of injectors for farm animals, the first five advantages are not implemented. In the developed device for vaccination, these advantages are realized due to the use of a linear motor of a new design.

\section{Conclusions}

The existing designs of devices for vaccination of farm animals do not allow automating the calculation and control of the issued doses of substance for injections.

The most promising type of plunger rod drive in injection devices is an electric drive, but existing devices are not able to realize the four typical stages of linear movement of the plunger rod.

All four typical stages of linear movement of the plunger rod can be realized by a linear motor. In addition, the linear motor allows one to adjust the duration of the injection process, control the depth of the needle penetration, vary the volume of the administered dose of the medicine, reverse the needle after injection, automatically fill the syringe with the next dose of the medicine.

\section{References}

1. S. Norn, P.R. Kruse, E. Kruse, On the history of injection, Dansk Medicinhistorisk Årbog, 34, 104113 (2006)

2. P. Wadham, Syringe pump design, Engin. in Med., 15(4), 171-174 (1986)

3. A. Jeanes, Zero-Stik-Safety Syringe: an automatic safety syringe, British J. of Nurs., 8(8) (1999) (Mark Allen Publ.), Retrieved from: https://doi.org/10.12968/bjon.1999.8.8.6633 
4. J.W. Ong, D.C.K. Chung, E.S. Lin, H.A. Abid, O.W. Liew, T.W. Ng, Syringe infusion pump with absolute piston displacement control, Rev. of Sci. Instr., 90(7) (2019), Retrieved from: https://doi.org/10.1063/1.5099271

5. M. Jafarzadeh, F. Farokhi, Design and construction of an automatic syringe injection pump, Pacific Sci. Rev. A: Nat. Sci. and Engin., 18(2), 132-137 (2016)

6. I. Saidi, L. El, A. Ounip, M. Benrejeb, Design of an Electrical Syringe Pump Using a Linear Tubular Step Actuator, Int. J. of Sci. and Techn. of Autom. Control \& Comp. Engin. IJ-STA, 4(2), 1388-1401 (2010)

7. I.N. Lei, S. Wen, Z. Wu, Q. Xu, Design and testing of a micro-syringe pump driven by piezoelectric actuator, Proc. of Int. Conf. Advanced Robotics and Mechatronics (ICARM 2016) (18-20 Aug. 2016, Macau, China), pp. 219-224

8. M.S.V. Appaji, G.S. Reddy, S. Arunkumar, M. Venkatesan, An 8051 Microcontroller based Syringe Pump Control System for Surface
Micromachining, Proc. Mat. Sci., 5, 1791-1800 (2014)

9. B. Wijnen, E.J. Hunt, G.C. Anzalone, J.M. Pearce, Open-source syringe pump library, PLoS ONE, 9(9) (2014), Retrieved from: https://doi.org/10.1371/journal.pone.0107216

10. M.R. Islam, R. Zahid Rusho, S.M. Rabiul Islam, Design and implementation of low cost smart syringe pump for telemedicine and healthcare, Proc. of 1st Int. Conf. on Robotics, Electrical and Signal Processing Techniques (ICREST 2019) (10-12 Jan. 2019, Dhaka, Bangladesh), pp. 440-444

11. G.V. Nikitenko, V.A. Grinchenko, Linear motor, Patent RF, no. 2370874 МПК8 H 02 K 33/12 (Linear motor applicant and patent holder Stavropol State agrarian university), no. 2008112342/09 (20 October 2009)

12. V.A. Grinchenko, Justification of the basic design of a linear electric motor, ISJ Theor. \& Appl. Sci., 11(7), 58-60 (2013), DOI: http://dx.doi.org/10.15863/TAS.2013.11.7.9 